\title{
L'administration communale dans le royaume de Westphalie
}

\section{Nicola Peter Todorov}

\section{(2) OpenEdition \\ 12 Journals}

Édition électronique

URL : https://journals.openedition.org/ahrf/8573

DOI : 10.4000/ahrf.8573

ISSN : 1952-403X

\section{Éditeur :}

Armand Colin, Société des études robespierristes

\section{Édition imprimée}

Date de publication : 1 mars 2007

Pagination : 113-137

ISSN : 0003-4436

\section{Référence électronique}

Nicola Peter Todorov, «L'administration communale dans le royaume de Westphalie », Annales

historiques de la Révolution française [En ligne], 347 | janvier-mars 2007, mis en ligne le 01 mars 2010, consulté le 23 avril 2022. URL : http://journals.openedition.org/ahrf/8573 ; DOI : https://doi.org/ 10.4000/ahrf.8573

Ce document a été généré automatiquement le 23 avril 2022.

Tous droits réservés 


\title{
L'administration communale dans le royaume de Westphalie
}

\author{
Nicola Peter Todorov
}

Après la défaite de la quatrième coalition, en 1807, Napoléon créa le royaume de Westphalie dont l'organisation devait être calquée sur celle de la France. Le transfert du modèle administratif français dans les pays d'Europe conquis par les armées françaises à l'époque de la Révolution et de l'Empire intéresse l'historiographie depuis longtemps. L'historiographie a généralement considéré que le transfert du modèle français dans les pays européens non transformés par une révolution s'était heurté aux résistances des populations. Les différentes traditions historiographiques nationales ont évidemment pesé sur les approches de la question. Sous l'influence des théories de Max Weber, l'historiographie allemande des années 1980 et 1990 a fait du concept de bureaucratisation un paradigme central ${ }^{1}$. La modernisation de l'État et de la société est conçue comme l'œuvre de bureaucraties, c'est-à-dire d'administrateurs professionnels, disciplinés, rationnels et dotés d'une conscience de groupe $^{2}$. Il est clair que l'administration locale, celle des villes, villages et hameaux, n'ayant jamais été entièrement investie par des administrateurs de métier, n'était pas au cœur des préoccupations de cette historiographie ${ }^{3}$. L'administration par excellence était celle des échelons supérieurs. "C'est que seulement cette partie du service public [la très haute administration] obtenait la pleine jouissance de la sécurité juridique et des privilèges matériels et était de par sa fonction en mesure d'agir en tant que créateur politique $»^{4}$. Mais cette approche a également incité les historiens à adopter le point de vue de ces administrateurs professionnels, instruits et producteurs de l'essentiel des sources ${ }^{5}$. Les bureaucrates étaient en effet assez critiques à l'égard des administrateurs locaux.

Deux grands types de critiques ont été adressés aux réformes administratives réalisées, sous l'influence directe ou indirecte de Napoléon, au début du XIX siècle en Allemagne. La première critique était d'ordre pratique. On a insisté ainsi sur l'écart béant entre les ambitions des administrateurs professionnels, préfets, sous-préfets, instruits et inspirés de l'idéologie des Lumières et les réalités du terrain, où des masses surtout rurales, illettrées et superstitieuses, se seraient montrées peu compréhensives à l'égard 
des efforts déployés par les premiers. Les préfets auraient eu dû mal à trouver du personnel qualifié ${ }^{6}$ pour ces fonctions communales rebutantes ${ }^{7}$.

3 Le deuxième reproche était moins d'ordre pratique que politique. L'étatisation de l'administration locale, c'est-à-dire l'intégration formelle de l'échelon local dans une seule hiérarchie, aurait empiété sur l'autonomie et la liberté ancestrales des communes. On oppose ainsi le modèle communal français au modèle prussien, tel qu'il fut mis en vigueur par la réforme de Stein (Städteordnung) en 1808, et qui était caractérisé par une autonomie plus large des communes urbaines ${ }^{8}$. Cette mise en opposition paraît quelque peu étrange, car la réforme de Stein en Prusse ne touchait que les communes urbaines, alors que la majorité de la population vivait encore à la campagne... et restait soumise au régime seigneurial.

Ces deux critiques, déjà formulées par les contemporains, ont amené les gouvernements centralisateurs à reculer dans les décennies suivant la défaite de Napoléon ${ }^{9}$. Dès la période impériale, les administrations intermédiaires ont cherché à persuader les gouvernements à renoncer à une administration locale investie par des non-professionnels et à créer des circonscriptions plus grandes qu'administreraient des agents professionnels ${ }^{10}$. Le problème du bon choix de la taille des circonscriptions administratives n'était pas inconnu aux administrateurs venus réorganiser l'Allemagne. L'expérience, avortée ${ }^{11}$, des municipalités cantonales incitait le gouvernement impérial à s'opposer avec fermeté à la résurrection d'un nouvel échelon administratif ${ }^{12}$. Cette obstination à préserver l'existence des communes aurait dû faire deviner que derrière des considérations techniques se cachaient des enjeux sociaux, voire politiques.

5 Napoléon avait misé sur l'effet positif d'une nouvelle administration pour séduire les populations des territoires conquis. N'avait-il pas déclaré à son frère Jérôme : «Quel peuple voudra retourner sous le gouvernement arbitraire prussien, quand il aura goûté les bienfaits d'une administration sage et libérale ? $\aleph^{13}$. Encourageant le roi novice à persévérer dans l'effort réformateur, il lui écrit : « Cette manière de gouverner sera une barrière plus puissante, pour vous séparer de la Prusse, que l'Elbe, que les places fortes et que la protection de la France ».

6 L'étude des enjeux et du fonctionnement de l'administration locale à l'époque napoléonienne est susceptible de nous éclairer non seulement sur les rapports entre les Français et les populations locales mais aussi sur le développement de l'État. Nous nous proposons ici d'analyser les changements institutionnels, la composition des corps d'administrateurs et le fonctionnement de l'administration communale dans un État vassal de l'Empire français. Le cadre de notre étude porte sur un département emblématique du royaume de Westphalie, celui de l'Elbe entre 1807 et 1813.

Des communes mises sous tutelle ?L'administration communale avant 1808

7 La première conséquence de l'application du modèle communal français est l'abolition de l'inégalité statutaire entre les communes urbaines et les communes rurales.

8 Avant 1808, la circonscription administrative de base est généralement l'unité de peuplement. Le statut juridique des villes et celui des villages se distinguent nettement. Les villes dites immédiates sont directement subordonnées aux autorités royales de leur province. Un « conseiller des tailles » surveille en général une dizaine de villes. Le roi de Prusse nomme les bourgmestres et les autres membres du conseil municipal (Magistrat). Le gouvernement de Berlin contrôle aussi directement la comptabilité des villes. Le contrôle de l'administration urbaine par les autorités royales de la province 
s'étend jusqu'à la fixation des salaires des principaux agents. Ainsi, en 1801, le conseil de la ville de Magdebourg se voit refuser sa demande de pouvoir augmenter d'une centaine de Thalers, aux frais de la caisse communale, bien remplie, les rémunérations de ses membres ${ }^{14}$.

Les villes constituent une circonscription judiciaire et administrative. Le tribunal municipal représente la première instance pour ses habitants. L'administration royale réglemente aussi la division du travail des conseils municipaux. Le conseiller des tailles élabore ainsi les instructions pour les différents membres du conseil. La délimitation des attributions est même plus précise que celle des différents membres des collèges provinciaux ${ }^{15}$. Autrement dit, la division disciplinaire du travail au détriment du principe géographique, qui caractérise les réformes administratives du début du XIX siècle, ne prend pas nécessairement son départ dans les administrations supérieures. Ainsi, une petite ville comme Schönebeck est administrée par un premier bourgmestre qui dirige le travail de l'administration municipale, qui reste toutefois une instance collégiale. Le second bourgmestre s'occupe de la justice. Enfin, les attributions du camérier sont importantes et leur description minutieuse - le descriptif de ses attributions comporte 17 paragraphes -, tout autant que celles des premier, deuxième, troisième et quatrième conseillers municipaux. La comptabilité des villes est d'ailleurs soigneusement vérifiée par les chambres administratives provinciales. Les comptes des villes dont les recettes dépassent 1000 Thalers sont envoyés à Berlin.

Les villages et les bourgs dits médiats relèvent d'abord de la tutelle du seigneur, qui peut être le roi. La différence entre villes et campagnes est aussi de nature fiscale. Les villes paient pour l'essentiel l'accise, c'est-à-dire une sorte d'impôt indirect, alors que les villageois doivent s'acquitter d'une contribution foncière. La plupart des mille unités de peuplement - les villages et hameaux - sont administrées par un Schulze ou Richter nommé par le seigneur. La structure de peuplement est variée dans le département de l'Elbe. Les gros villages-tas caractérisent la Börde de Magdebourg, la société rurale y est très stratifiée. Les laboureurs, qui en forment le sommet représentent parfois seulement $5 \%$ ou moins des ménages. Dans la Vieille Marche au Nord, l'habitat est constitué par de petits villages ou hameaux, où, en revanche, la société rurale semble être beaucoup plus homogène.

La nouvelle administration communale

11 Le gouvernement westphalien uniformise les fonctions municipales. Toutes les communes sont administrées par un maire. En dehors du maire, il y a de nombreuses autres fonctions ou services municipaux. L'adjoint au maire joue le rôle de policier au village. Le gouvernement fait preuve d'une certaine souplesse pour respecter la volonté d'autonomie des communautés. Ainsi, on nomme dans les villages minuscules des adjoints au maire alors qu'à l'origine un seul adjoint était prévu pour les municipalités de moins de 2500 habitants. Cela répond au vœu des collectivités.

12 Le secrétaire de mairie, d'abord choisi par le maire, est nommé à partir de 1809 par le préfet, afin de le rendre moins dépendant du maire. En théorie, il doit y avoir un percepteur élémentaire dans chaque commune. Quand la commune collecte d'importantes sommes à titre de taxes communales, il y a parfois un percepteur communal particulier. En principe, le gouvernement interdit le morcellement de ces fonctions pour diminuer le coût de l'administration communale.

13 Pour la majorité des petites communes rurales, la nouveauté est la création du conseil municipal, c'est-à-dire d'un organisme clairement défini. Auparavant, il n'y avait que 
l'assemblée villageoise qui ne représentait en fait que les propriétaires du territoire communal. Désormais, le conseil municipal comprend huit membres dans les petites communes et le double dans les communes de plus de 2500 habitants.

À lire le règlement administratif du royaume de Westphalie, les attributions des conseils municipaux sont restreintes: ils doivent se réunir une fois par an, le 15 novembre, pour une période de 10 jours pour débattre du budget de la commune ${ }^{16}$. En dehors de cette réunion, le conseil municipal ne peut être convoqué qu'avec l'autorisation du préfet, sur avis motivé du sous-préfet ${ }^{17}$. Les conseillers doivent entendre l'état des recettes et des dépenses communales, répartir le bois, les pâtures et les récoltes de la commune, discuter des emprunts et des centimes additionnels nécessaires à la satisfaction des besoins communaux et répartir les travaux d'entretien ${ }^{18}$. En réalité, la législation ultérieure du royaume de Westphalie attribue bien d'autres fonctions aux conseils municipaux, notamment dans le domaine de la répartition des impôts et de l'estimation des ressources des contribuables. Par ailleurs, la correspondance administrative montre qu'en cas de besoin, l'activité du conseil municipal ne se limite pas à une courte période de dix jours dans l'année. Le décret royal du 7 novembre 1808 autorise le ministre de l'Intérieur à convoquer les conseils municipaux selon les besoins des communes ${ }^{19}$.

Déjà, le 23 novembre 1808, transmettant les décrets de nominations de ces conseils au préfet du département de l'Elbe, le ministre Siméon écrit à de Schulenbourg-Emden :

« En attendant je n'ai pas besoin de vous rappeler que ce n'était qu'à leur défaut seul que les maires étaient chargés par les lois de la sous-répartition des impôts. Comme votre département peut aujourd'hui, grâce à vos soins constants, jouir pleinement de l'ordre constitutionnel, cette opération délicate doit être laissée à la représentation communale.

Vous aurez donc attention quand la répartition aura été faite aux districts par le conseil général et aux communes par les conseils de district, de faire faire la sousrépartition dans les communes par les conseils municipaux. Vous tiendrez la main à ce qu'elle s'opère avec clarté et impartialité suivant les instructions que vous aurez reçues de mon collègue le ministre des Finances ${ }^{20}$.

La division communale westphalienne constitue une voie moyenne entre deux extrêmes précédemment expérimentés: d'un côté, le système des municipalités cantonales, qui a bafoué la volonté d'auto-gouvernement des collectivités villageoises, et celui du morcellement des municipalités selon les unités de peuplement: villages, hameaux, fermes isolées. Si bien des villages d'une certaine taille continuent à former une unité administrative, d'autres sont rattachés à des villages plus grands ou fusionnent avec eux. Là où la taille et le revenu d'une commune justifient la présence d'une mairie, elle lui est accordée. Dans les contrées d'habitat dispersé, en revanche, plusieurs petits villages et hameaux sont regroupés pour ne former qu'une seule commune. Le regroupement des unités d'habitat prend une grande ampleur dans le district de Salzwedel. Dans le canton de Diesdorf, 11 des 14 communes sont composées d'au moins 3 unités et 2 communes en ont 6 . Des cas où un maire administre 6 unités de peuplement existent également dans les cantons de Pollitz, Gardelegen municipal, Beetzendorf. Dans le canton rural de Gardelegen et dans celui de Brohme, il y a des communes de 7 hameaux et maisons isolées. Un des effets de la nouvelle division communale était l'abolition de l'autonomie administrative des anciens domaines seigneuriaux. 

plus de 5000 habitants, le roi de Westphalie se réserve la nomination de tous les maires, voire de tous les conseillers municipaux. Or, il y a 2840 communes dans le royaume ${ }^{21}$, et plus de 20000 conseillers municipaux.

d'abord n'envoyer que les budgets des communes de plus de 2500 habitants au ministère, puis, à partir de 1810 , les budgets de toutes les communes dont les recettes dépassent 2000 francs et surtout de toutes celles dont le déficit est supérieur à 100 francs. Le préfet et le sous-préfet n'ont donc plus qu'à donner leur avis au même titre que le maire et le conseil municipal. Ces budgets font l'objet de décrets royaux après l'avis du ministre de l'Intérieur ${ }^{22}$. Ainsi, en 1812, plus d'un tiers (200) des budgets communaux du département de l'Elbe sont arrêtés par le ministère ${ }^{23}$, alors qu'en 1800 , 10 seulement des 33 budgets des villes du duché de Magdebourg furent envoyés à Berlin: ceux dont les recettes dépassaient 1000 écus ( 3700 francs) et la réforme décentralisatrice de 1800 allait monter la barre à 2000 écus $(7400 \text { francs })^{24}$. Les comptes des communes rurales étaient éventuellement vérifiés par les juges seigneuriau $\mathrm{x}^{25}$. Les anciennes listes de tarification des sportules administratives prévoyaient d'ailleurs un tarif pour la révision d'un compte communal ${ }^{26}$. La préfecture westphalienne quant à elle vérifie la totalité des budgets communaux et ce travail régulier absorbe un certain temps. Au ministère, composé d'une quinzaine de personnes, un commis est spécialement chargé de la vérification des budgets ${ }^{27}$.

19 Sur le plan institutionnel, l'exportation du système communal français ne s'accompagne que dans une certaine mesure d'une mise sous tutelle des communautés locales. Le nouvel État détermine le nombre des agents municipaux tout en admettant certains aménagements. L'introduction d'un budget prévisionnel oblige les communes à limiter leurs dépenses. Mais celles-ci faisaient déjà, avant 1808, l'objet d'une surveillance par les autorités supérieures, essentiellement provinciales ou seigneuriales. Ce qui change après 1808, c'est l'autorité chargée du contrôle des communautés: de l'échelon provincial, la mission de surveillance passe à l'échelon central. La composition des différentes instances et leurs intérêts n'étaient pas les mêmes, car la description des attributions réglementaires ne peut rendre suffisamment compte du fonctionnement d'une administration.

Des administrateurs locaux incapables ?Un certain renouvellement du personnel local Les plaintes des préfets et des sous-préfets au sujet de la qualité des administrateurs municipaux, notamment ceux des petites communes rurales, sont un fait bien connu dans l'Empire français et, dans les États satellites, la Westphalie ne fait guère exception. Après quatre années de réformes, le sous-préfet du district de Salzwedel, L. de Westphalen, écrit, certes pour justifier la nécessité d'un traitement et de frais de bureau plus élevés :

«Les maires des communes rurales dans le district de Salzwedel ayant dû être choisis parmi la classe des paysans, ne sont pas assez instruits et ne possèdent pas l'habileté nécessaire pour s'acquitter de leurs fonctions et principalement de la gestion des affaires, qui ont rapport à la répartition des contributions sous la direction et les instructions immédiates des maires de canton; ils n'ont d'ailleurs nullement la faculté de donner des renseignements par écrit.[...] il est impossible à l'homme le plus habile et le plus actif et laborieux de suffire à l'immense besogne de toutes les écritures relatives non seulement aux affaires courantes très nombreuses et à la correspondance très étendue à entretenir avec les différentes autorités et particulièrement avec les agents des contributions, mais aussi à la confection des 
listes communales de conscription, des budgets, des états de contributions personnelles [...] des rôles de répartition des différentes contributions départementales et communales; des liquidations mensuelles des frais d'étapes et des frais de prison, des procès-verbaux à dresser sur les délibérations des conseils municipaux pour obtenir l'autorisation à plaider, etc., etc. [...] $»^{28}$.

21 Le corps des administrateurs locaux westphaliens est pourtant plus restreint que celui des anciens officiers villageois, notamment dans les régions à habitat dispersé en raison du regroupement des petits villages et hameaux en une seule commune. Les 1000 unités de peuplement sont regroupées en 474 communes $^{29}$. On doit donc en principe disposer d'un vivier d'administrateurs expérimentés. Nous avons dénombré ainsi, à partir des listes de candidats pour les conseils municipaux, 148 anciens Schulze vivant toujours dans les villages du district de Salzwedel et 101 dans ceux du district de Stendal. Même quand on déduit les Schulze relégués au poste d'adjoint, 63 anciens officiers villageois du district de Salzwedel et 79 de celui de Stendal ne sont pas repris par l'administration westphalienne ${ }^{30}$. Malgré la réduction du nombre de postes d'administrateurs locaux, les maires ne sont pas systématiquement recrutés dans le vivier des anciens officiers villageois. Seulement 175 des 474 premiers maires westphaliens du département de l'Elbe se trouvaient avant 1808 à la tête de leur collectivité locale ou de l'une de ses parties. Autrement dit, 299 communes du département de l'Elbe reçoivent un chef n'ayant pas encore exercé la charge de bourgmestre, de Schulze ou Richter. Le remplacement, en juillet 1808, des maires démissionnaires fraîchement nommés ne permet le retour que de 14 des anciens Schulzen ou bourgmestres. Le renouvellement massif du personnel de l'échelon administratif inférieur est donc bien réel.

22 La situation des districts de Neuhaldensleben et de Magdebourg, où la continuité personnelle est importante, contraste avec celle des districts de l'ancienne Vieille Marche. Cependant, même dans les deux premiers districts, 40 \% des villages sont dotés d'un nouveau chef. La mise à l'écart des anciens officiers seigneuriaux - Schulzen ou Richter - est d'autant plus significative dans la Vieille Marche que les communes y sont composées de plusieurs villages et hameaux et que dans chacune d'elles habitent alors plusieurs anciens préposés de villages.

23 Le renouvellement du personnel administratif local s'explique partiellement par la tentative des élites traditionnelles de contrôler directement un échelon administratif qu'elles n'occupèrent pas jusqu'alors. Dans le département de l'Elbe, comme dans le royaume de Westphalie, au moins au début du règne de Jérôme-Napoléon, des tentatives individuelles et collectives de la noblesse d'occuper les postes de l'administration locale et cantonale, sans doute avec l'appui de nobles appartenant au gouvernement, sont indéniables. Ainsi de Wolfradt, ministre de l'Intérieur du royaume de Westphalie à partir de 1809, nous dit dans ses mémoires :

«La plupart des nobles suivaient mon conseil d'accepter eux-mêmes les postes de maires dans leurs communes, ils y furent nommés de préférence " ${ }^{31}$.

24 Les anciens seigneurs jugent sans doute nécessaire d'être présents personnellement dans les institutions communales en raison de la disparition de l'ancien cadre seigneurial ou bailliager. 
Les maires nouveaux et le noyautage seigneurial

\begin{tabular}{|c|c|c|c|c|}
\hline District & Magdebourg & Neuhaldensleben & Stendal & Salzwedel \\
\hline Maires nobles & 8 & 8 & 27 & 3 \\
\hline Maires fermiers & 10 & 7 & 14 & 6 \\
\hline Total des précédents & 18 & 15 & 41 & 9 \\
\hline Maires « nouveaux » & 42 & 45 & 101 & 111 \\
\hline
\end{tabular}

La tentative de noyautage de l'administration communale par les partisans de l'ancien ordre social, si indéniable qu'elle soit, ne connait dès le début qu'un succès limité. Le grand nombre de communes empêche la noblesse d'occuper tous les postes de maire. Les fermiers ne sont pas non plus suffisamment nombreux pour assumer la fonction de maire dans la totalité des communes.

Ce constat vaut encore davantage pour les autres fonctions communales: le poids numérique des grands propriétaires fonciers et de leurs fermiers dans les conseils municipaux est encore beaucoup plus faible que dans le corps des maires et ne reflète que leur poids numérique dans la société. Leur pouvoir réel au sein des conseils municipaux reste probablement beaucoup plus grand mais dépend sans doute très fortement de la composition du conseil municipal de chaque commune ainsi que de la politisation des habitants. Ainsi dans quelques communes du district de Stendal, un propriétaire noble, en qualité de maire ou de simple conseiller municipal, peut faire face à 7 micro-propriétaires ou journaliers au sein du conseil, comme c'est surtout le cas dans les villages de la vallée de l'Elbe, où la paysannerie moyenne est parfois complètement absente.

La composition sociale du corps des fonctionnaires locaux traduit bien souvent la géographie sociale. Ainsi, dans les communes du Nord-Ouest, où la population rurale est relativement homogène - les laboureurs représentent parfois $50 \%$, voire la totalité des feux - la paysannerie moyenne investit la plupart des fonctions communales, du maire au secrétaire de mairie en passant par le percepteur élémentaire. En revanche, au Sud-Est, dans le district de Magdebourg, où la population rurale est très stratifiée, le poste de maire est souvent occupé par un laboureur, alors que le percepteur élémentaire est souvent un petit ménager, augmentant ses revenus par cette activité complémentaire. Quant au conseil municipal, il représente les principales catégories sociales des collectivités. Dans $10 \%$ des communes du district de Stendal, les laboureurs sont les seuls à investir le conseil municipal, ce qui ne correspond pas toujours à la composition sociale des principaux groupes de propriétaires paysans. Si les marchands et artisans sont présents, bien que faiblement, dans la plupart des conseils municipaux, ils n'occupent que rarement les postes de maires dans les communes rurales. En revanche, ils dominent les conseils des villes. Dans une ville de 5000 habitants comme Stendal, le conseil municipal est formé par cinq marchands, cinq artisans, deux fabricants et quatre personnes touchant un salaire.

La réaction seigneuriale 
28 Le préfet et les sous-préfets, généralement de connivence avec les anciennes élites seigneuriales, cherchent alors à obtenir du gouvernement la modification de la division communale par le rattachement de certaines communes à d'autres ou par la fusion de plusieurs petites communes. L'analyse détaillée de ces propositions révèle en général l'objectif, inavoué, de reconstituer le cadre territorial des anciennes seigneuries, formellement supprimées. Mais généralement ces projets essuient un refus net de la part du ministre de la Justice et de l'Intérieur, Siméon. Le gouvernement westphalien est donc aussi attaché à l'autonomie des petites communes que le gouvernement impérial.

Selon Siméon, la création des maires de canton est d'abord une mesure ponctuelle, qui est étendue ensuite à l'ensemble du royaume. Le 14 novembre 1808, exactement sept mois après la nomination de la plupart des maires de commune, Siméon s'adresse au préfet de l'Elbe pour l'inviter à proposer des candidats pour les postes de maire de canton, une mesure souhaitée depuis plusieurs mois par les autorités départementales. Le gouvernement est bien conscient des ambiguïtés de cet écart par rapport au modèle français. Les compétences des maires de canton sont donc assez vaguement délimitées. Aux yeux du gouvernement, il ne s'agit absolument pas de créer un échelon bureaucratique supplémentaire. Les mairies de canton ne sont pas dotées d'un secrétaire réglementaire, c'est-à-dire d'un fonctionnaire nommé par le roi. Wolfradt, le ministre de l'Intérieur, précise bien au préfet de l'Elbe, la situation ambiguë du maire de canton :

«S'il existait des secrétaires dans les mairies de canton, leur nomination et leur remplacement appartiendraient à Sa Majesté comme celui des Maires et des Secrétaires de Mairies dans les communes d'une population supérieure. Mais il n'a pas été accordé de secrétaires aux mairies de canton, parce que ces magistrats dont l'institution est temporaire, et non constitutionnelle n'ont réellement que l'impulsion et des conseils à donner à leurs collègues, le plus souvent de vive voix, et ne sont pas proprement chargés d'administrer, mais d'éclaircir et de diriger l'administration des communes. En chargeant le maire le plus intelligent du canton de guider les autres, on n'a point entendu créer de nouvelles fonctions, mais faciliter la marche de l'administration municipale dans les commencements d'un système nouveau ${ }^{32}$.

30 En tout état de cause, les quatre administrateurs des districts du département de l'Elbe en 1808, à savoir le préfet et les trois sous-préfets, profitent de l'installation des maires de canton pour proposer de préférence des nobles et des fermiers royaux. Si, à l'échelle du département, les nobles, avec plus d'un tiers des personnes, ne constituent pas la majorité des maires de canton, ils fournissent $90 \%$ des maires de canton du district de Stendal, alors qu'au niveau communal la noblesse ne représente qu'un peu plus d'un cinquième des maires de ce district. Cette prédominance reflète l'implantation locale de la noblesse. Dans la partie de la Börde magdebourgeoise, qui forme le district de Magdebourg, la noblesse est moins présente. Mais même à l'échelle départementale, la noblesse est plus représentée dans le corps des maires de canton que dans celui des maires de commune. La nomination de nombreux fermiers conduit à une domination nette du corps des maires de canton par les classes opposées à l'abolition des redevances sans rachat.

31 Ne faudrait-il pas plutôt se demander si l'incapacité des masses rurales à remplir les fonctions administratives n'était pas un argument commode utilisé par un corps préfectoral acquis aux idées traditionnelles pour imposer un autre encadrement, permettant mieux de contrôler des collectivités récalcitrantes? Le gouvernement 
westphalien ne tient d'ailleurs pas trop compte de ces lamentations. Ainsi, lors de la révision des nominations en 1810, le sous-préfet de Stendal, le comte de SchulenbourgBodendorf, propose de destituer 49 des 120 maires par le biais de fusions de communes, sans compter les autres remplacements. Or, le gouvernement reconduit 44 de ces maires et ne supprime aucune commune. Sur les 6 maires remplacés : 4 sont des nobles et le cinquième un grand fermier. Le gouvernement se montre donc aussi inflexible que celui de l'Empire dans le département belge de la Dyle, étudié par Tihon, quand il s'agit de limiter l'emprise des anciennes élites sur les sociétés rurales.

Les villes, en tous cas, disposent d'administrateurs professionnels. Dans les villes du duché de Magdebourg et de la Vieille Marche, il y avait, en 1807, une centaine d'administrateurs municipaux: 68 d'entre eux disposaient d'une fortune foncière, le plus souvent leur maison. Plus de la moitié de ces administrateurs déclarent dans les questionnaires que devaient remplir les personnes désirant intégrer le service westphalien avoir suivi une formation au-delà de l'enseignement élémentaire, 38 ont obtenu une formation universitaire, généralement à l'université de Halle ${ }^{33}$.

L'investiture des postes communaux constituait donc manifestement un enjeu pour les élites traditionnelles comme pour les autres administrés. En principe, l'administration locale westphalienne peut s'appuyer sur un personnel issu de l'ancienne administration villageoise et urbaine ou des mêmes couches sociales. Rejeter les critiques des préfets et sous-préfets issus ou proches des élites ci-devant privilégiées comme trop intéressées pour être prises au sérieux par l'historien nous exposerait cependant au reproche d'une vision partiale, si l'analyse excluait le fonctionnement de cette administration.

Le fonctionnement de l'administration communaleUne participation relativement volontaire des habitants

Pour mesurer les difficultés réelles de l'adoption du modèle communal français en Westphalie, il est nécessaire de le voir à l'œuvre. En avril 1808, lorsque les maires sont nommés, les refus d'accepter la fonction ou les démissions précoces sont relativement limités. En juillet 1808, moins de $10 \%$ des communes n'ont pas encore leur maire. Dans d'autres départements, ce taux est encore plus bas ${ }^{34}$, mais le taux des mairies encore vacantes dans le département de l'Elbe varie de $1,9 \%$ dans le district de Neuhaldensleben à $15 \%$ dans celui de Stendal. Les fonctionnaires sont nommés par un décret.

Entre 1808 et la fin de l'année 1812, 271 maires sont remplacés dans les 450 communes qui ont appartenu au département de l'Elbe pendant toute la période westphalienne. Mais le taux de renouvellement varie dans le temps. Au cours de la première année, moins de $11 \%$ des maires sont remplacés. Plus de $30 \%$ des communes reçoivent un nouveau maire en raison de la révision des nominations que le gouvernement réalise en 1810. Le taux retombe à un peu plus de $10 \%$ en 1811 et à $8 \%$ de l'ensemble des maires en 1812. Le personnel des fonctionnaires municipaux semble donc se stabiliser durant la période westphalienne. Ces chiffres cachent pourtant d'importants écarts spatiaux : 208 communes ( $46 \%$ ) n'ont eu qu'un seul maire durant la période westphalienne, 182 ( $40 \%)$ en ont eu 2, alors que $55(12 \%)$ en ont vu se succéder 3 . Il y a quelques cas extrêmes, assez rares ( $1 \%)$, où durant la courte période napoléonienne, 3 communes sont administrées par 4 maires et 2 par 5 maires.

36 Avant 1808, la situation des officiers communaux était déjà ambiguë. Normalement, la charge n'était pas limitée dans le temps, le renouvellement des agents assez rare. Les témoignages sur l'attrait de la fonction avant 1808 sont cependant contradictoires. 
Certains paragraphes du règlement pour les villages du duché de Magdebourg, élaboré en 1805 par les conseillers provinciaux nobles et les officiers de la chambre provinciale, semblent indiquer que la charge du préposé de village (Dorfvorsteher - Schultze ou Richter) n'était guère enviable: «Un membre de la communauté ne peut refuser la charge de préposé de village ou de juré que l'autorité lui a attribuée que pour des raisons qui l'empêcheraient d'assumer une tutelle $»^{35}$. ( $\left.\$ 5\right)$. D'ailleurs, des clauses analogues se trouvent dans les statuts des villes.

D'un autre côté, dans certains cas, l'investiture de la première charge communale donne lieu à des contestations notamment au sujet de l'appartenance de l'officier villageois à telle ou telle catégorie d'habitants. Dans les villages, les laboureurs acceptent mal qu'un petit paysan (Kossathe) soit nommé officier seigneurial ${ }^{36}$. En principe, ce n'est guère différent dans les petites villes. En 1806, juste après l'occupation française, les "petits bourgeois » de la ville de Frohse se plaignent des inégalités de la répartition des soldats à loger et en rendent le bourgmestre Kaufmann responsable. Frohse ne comporte que huit fermes paysannes et une centaine de petites maisons. Chaque petit bourgeois de la ville doit héberger trois ou quatre soldats français. Le batelier Samuel Reichmann, un ancien cuirassier des gardes du corps, et le maçon Jean-Jacques Bunge organisent alors une réunion des petits bourgeois à l'hôtel de ville et chargent le garde champêtre Naucke de sonner le tocsin. Lorsque tout le monde est réuni, ils font traîner violemment le bourgmestre devant l'assemblée pour lui faire comprendre leur point de vue. Après avoir été menacé, il est relâché après avoir promis de présenter un plan clair pour le logement des troupes ${ }^{37}$. Dans ces petites villes, qui deviendront les faubourgs des villes industrielles du XIX ${ }^{e}$ siècle, le petit artisanat et les journaliers se montrent souvent assez revendicatifs.

D'une manière générale, la sous-répartition des charges publiques a aussi dû être un des moments forts de la vie publique communale sous le gouvernement westphalien. Elle confère à ceux qui en sont chargés un pouvoir sur les autres membres de la commune, ce qui doit rendre la fonction de conseiller municipal attrayante. Si l'on ne dispose guère de témoignages de la plupart des communes du département de l'Elbe sur l'attrait de cette fonction, des réclamations quant à la juste distribution des charges publiques et à la composition des conseils sont loin d'être rares.

Le maire de Schleibnitz donne sa démission parce que, notamment, les Kossathen, refusant de supporter des charges communales ancestrales, lui rendent la vie difficile ${ }^{38}$. En février 1809, donc après la nomination des conseillers municipaux, le maire Kloetze de Hilligendorf écrit au préfet :

«Sur la demande des Kossathen d'ici, qui sont au nombre de 22 et dont aucun n'a été nommé au conseil municipal, j'ai l'honneur de prier Votre Excellence d'opérer un changement, car 3 des laboureurs y ont été nommés. À mon sens, il y en a un qui pourrait céder sa place à l'un des ménagers.

Je prie votre Excellence d'autoriser cette modification gracieusement et ose proposer le Kossathe Christophe Bennecke, auquel, en revanche, le cultivateur Christian Engel devrait céder sa place [...] $\gg^{39}$.

La composition sociale des différentes institutions communales n'est donc pas indifférente aux habitants. Mais ce dernier cas suggère aussi qu'au sein des petites communes les jeux politiques n'étaient pas absents. Le maire cherche peut-être à avoir au conseil municipal un contre-pouvoir que représentent les ménagers face aux laboureurs. Les anciens seigneurs qui aspirent à contrôler leurs anciens dépendants en se faisant nommer maire cherchent également à diviser la communauté locale en 
jouant sur les divergences d'intérêts des différents groupes. Ainsi le baron d'Angern, l'ancien président de la chambre administrative, qui a ardemment défendu les intérêts des grands propriétaires fonciers en tant qu'administrateur de la province, retourne après la défaite de la Prusse dans son domaine de Sülldorf, aux portes de Magdebourg. Le décret royal du 13 avril 1808 le nomme maire de sa commune. Lorsque les laboureurs se plaignent à la préfecture de la répartition des corvées publiques de transports militaires, contestant l'exemption des ménagers, le maire, grand propriétaire, prend le parti de ces derniers. Interrogé par le préfet, le maire donne aussi son avis sur les possibilités d'astreindre les nobles à ces services. Au cas où ceux-ci devraient participer à ces charges publiques, ils devraient être chargés proportionnellement moins que les laboureurs, estime-t-il. Le laboureur aurait acheté son bien moins cher en raison des charges publiques. Il entretiendrait plus de chevaux par surface arable que le noble en raison de ces corvées et serait donc mieux en mesure de fournir les services réclamés ${ }^{40}$.

41 Ce qui est intéressant dans cette affaire n'est ni son argumentation, qui laisse perplexe, ni la réaction du préfet, qui la trouve pertinente ${ }^{41}$. L'intérêt réside dans le soutien qu'apporte le grand propriétaire aux petits ménagers de la commune. Ne s'agit-il pas de monter ces derniers contre les laboureurs moyens selon la devise : diviser pour mieux régner? La réussite de ces jeux dépend aussi de l'attitude des autorités préfectorales. Or, avant la nomination du Français Bercagny en 1812, la préfecture est acquise aux intérêts des anciens seigneurs. Le préfet, le comte de Schulenburg-Emden réussit parfois à faire écran entre les collectivités et le gouvernement. Lorsque, par exemple en décembre 1811, le conseil municipal de la petite ville de Gross-Salze s'oppose à la nomination d'un propriétaire foncier à la fonction de maire ou d'adjoint, comme le propose le maire démissionnaire, un propriétaire, noble lui aussi, il n'est pas écouté, et la personne à laquelle les conseillers sont hostiles est nommée adjoint au maire ${ }^{42}$.

42 Mais ces litiges au sujet de la répartition des charges publiques régulières datent pour l'essentiel du début du gouvernement westphalien. Leur fréquence a dû diminuer au cours de la période, car le gouvernement s'attache à contrôler de près les finances communales.

Surveiller pour protéger

La surveillance des budgets communaux n'est en effet pas purement formelle. Malheureusement, seul un petit nombre de budgets détaillés a été conservé. Mais les quelques budgets dont nous disposons nous indiquent que le conseil municipal propose parfois pour la fonction de maire un salaire plus bas que celui proposé par le maire. Et fréquemment, le ministre ou le préfet réduisent encore ces dépenses. Ainsi, dans les 11 communes du canton d'Eichenbarleben, cinq des conseils municipaux proposent en 1812 une somme plus basse pour le traitement du maire que ne l'ont fait les premiers fonctionnaires des communes eux-mêmes ${ }^{43}$. Le gouvernement semble avoir uniformisé le traitement des fonctionnaires municipaux. Un relevé des maires en place à la fin du règne de Jérôme et de leur traitement montre que dans les communes rurales les maires touchent 40 francs par $\mathrm{an}^{44}$.

Malgré le regroupement de plusieurs localités sous l'administration d'un maire, les communes à plusieurs composantes présentent généralement des déficits budgétaires. Si d'un côté, l'inégale richesse des communautés villageoises peut expliquer l'absence totale de recettes de nombreuses communes, c'est souvent le regroupement des villages qui est à l'origine du déficit. Les nouvelles communes ne font que se superposer aux anciennes communautés d'exploitation. Les collectivités locales sont en effet libres 
d'apporter leurs biens communaux à la nouvelle commune. Quand elles préfèrent la gestion distincte des biens communaux anciens et des biens de la commune westphalienne, cette dernière est sans propriété et doit couvrir ses dépenses en levant un impôt communal. Le poids de cet impôt supplémentaire est cependant assez léger parce qu'il est réparti sur plusieurs villages alors que les dépenses plafonnent assez vite en raison de leur contrôle multiple. D'ailleurs, en cas de gestion distincte des biens de la commune et de l'ancienne communauté villageoise, le gouvernement vérifie les deux budgets.

D'un autre côté, le ministre intervient dans le mode de répartition des charges communales. Les conflits au sujet de la sous-répartition des taxes levées pour payer le déficit des budgets communaux sont relativement rares. Ainsi, les petits ménagers du village de Löderburg s'adressent à la préfecture pour se plaindre du partage des charges effectué par le conseil municipal pour éponger le déficit de l'année $1809^{45}$. Celui-ci doit désormais se faire selon le pied de la contribution foncière et le Ministère de l'Intérieur vérifie son application au même titre que les dépenses communales ${ }^{46}$. Ce principe de répartition est aussi appliqué aux taxes que doivent verser les communes à l'administration forestière pour la gestion des bois communaux. Le choix de la contribution foncière comme base de répartition de ces déficits fait retomber ces charges sur les propriétaires. L'intégration des domaines dans les communes allège ainsi le coût de l'administration communale pour la majorité des habitants. Globalement très hostile à la fusion des communes, le gouvernement, en 1813, sépare une commune pour fusionner la composante la plus pauvre avec un grand domaine pour lui rendre les taxes communales plus légères. ${ }^{47}$

L'adoption d'un mode de répartition par le gouvernement est plus difficile lorsqu'il s'agit de charges irrégulières ou de charges en nature comme le cantonnement de militaires ou la livraison de chevaux. Ces charges dépendent fortement de la conjoncture militaire et se multiplient à la fin du règne de Jérôme. Ainsi, les événements politiques ont dû avoir une incidence sur l'intérêt que représentaient les charges communales. En 1813, le maire de canton de Sudenbourg écrit au préfet :

«[...] Sous les circonstances actuelles de la dite commune [celle de Cracau près de Magdebourg, assiégé] où déjà depuis un certain temps beaucoup de militaires sont cantonnés, les occupations du maire se sont multipliées concernant les hébergements, les réquisitions militaires et fournitures. Il est donc d'autant plus nécessaire qu'un adjoint lui assiste [...] Plusieurs des habitants le souhaitent d'autant plus que le dit Cornelius [le maire] appartient à la classe des Kossathen, alors que les laboureurs pensent que lors de la distribution des charges de logements, de fournitures militaires et autres, il ne pourra pas avoir assez égard aux conditions des laboureurs $»^{48}$.

Et le maire de canton de proposer, sur la demande des habitants de Cracau, que quelqu'un de la classe des laboureurs soit adjoint au maire. Aussi ne faut-il guère s'étonner de l'intérêt qu'ont les différents groupes de la paysannerie à être représentés au conseil municipal. Mais avant comme après 1806 , le travail administratif comporte aussi ses désagréments.

Un travail rebutant?

Les villes jouissent malgré leur taille d'une moindre indépendance que beaucoup de communautés rurales. Avant comme après 1808 , les administrateurs urbains se voient confrontés à la présence de puissantes administrations supérieures et ne constituent pas de ce fait le sommet de la hiérarchie sociale de la ville. Ainsi, à Magdebourg, il y 
avait en 1806, 18 administrateurs municipaux contre 67 administrateurs royaux prussiens dont les traitements étaient beaucoup plus élevés.

Les frictions entre les citadins et les commandants des garnisons prussiennes sont un fait bien connu ${ }^{49}$. Avant 1806, les empiètements des chefs de garnison ne se limitaient pas à des mauvais traitements ou des injures. Ainsi, le commandant de Schönebeck s'arrogeait le droit d'enfermer les bourgmestres dans la prison militaire et de les dénoncer aux autorités provinciales à chaque fois qu'il recevait des plaintes sur divers sujets, et notamment la police des grains et de la bière. Si le fait qu'un bourgmestre fut traîné, en robe de nuit, car les soldats ne lui avaient pas laissé le temps de s'habiller, devant le chef de garnison témoigne effectivement du peu de respect des autorités militaires face aux civils dans l'État prussien, les enjeux sociaux étaient parfois très complexes. Ainsi, le commandant, noble, accusait les administrateurs de la ville de ne représenter que la confrérie des brasseurs et boulangers et de s'enrichir aux dépens des pauvres de la ville et des soldats de la garnison. Dans les villes prussiennes, la population militaire était en effet importante. Ainsi, dans le faubourg de Frohse, elle représentait en 1805, plus de $16 \%$ des citadins ${ }^{50}$. En même temps, le commandant veillait au respect des règlements interdisant aux citadins de s'approvisionner directement à la campagne auprès des paysans ${ }^{51}$. Seuls les propriétaires nobles et les grands fermiers royaux avaient le privilège de vendre leur récolte sur place à des citadins venus à la campagne ${ }^{52}$. La chambre administrative provinciale ne s'intéressait d'ailleurs guère aux plaintes du conseil municipal et il fallait l'intervention $\mathrm{du}$ roi de Prusse pour que le commandant fût contraint de cesser, très partiellement, d'empiéter sur les prérogatives des administrateurs municipaux. Mais en raison de ces tracasseries, il n'est guère étonnant que les charges municipales ne fussent pas forcément plus recherchées que dans les villages. Sous le gouvernement westphalien, on cherche à améliorer les relations entre les militaires et les civils en nommant un Français préfet dans un département à forte présence militaire.

50 Le gouvernement westphalien demande à ses fonctionnaires communaux un certain nombre de tâches administratives et nous avons vu plus haut l'énumération qu'en a faite le sous-préfet de Salzwedel. Mais, d'abord, ces travaux ne sont pas une invention napoléonienne. En 1776, les habitants du village de Brumby s'étaient ainsi opposés à la nomination d'un parent du fermier royal au poste d'officier villageois : celui-ci serait illettré et donc tout à fait incapable de lire la gigantesque correspondance administrative ${ }^{53}$. Les statistiques napoléoniennes sont différentes mais pas nécessairement plus exigeantes que celles qui les précédaient. Ensuite, l'abondance des travaux administratifs et le désagrément qu'ils provoquent dépendent des conjonctures politique et militaire. Le cantonnement des militaires, leur approvisionnement et les réquisitions rendent en effet la vie difficile aux maires comme à leurs prédécesseurs. La géographie des démissions et destitutions de maires est en effet quelque peu surprenante. Les communes qui voient se succéder plusieurs maires durant la période westphalienne forment parfois des zones allongées. Or, dans certains cas, notamment celui du district de Salzwedel, ces bandes suivent le tracé du réseau routier. Enfin, les «maires» prussiens nommés après 1813 ou tout simplement laissés en place, sont curieusement bien capables de gérer une "paperasse » volumineuse, dont témoignent les inventaires de ces Schulzen ${ }^{54}$.

51 Le transfert de l'administration communale française dans une partie de l'Allemagne comportait un certain nombre de modifications institutionnelles, comme 
l'uniformisation des administrations, la centralisation des nominations et de la surveillance budgétaire et le retrait corrélatif de ces prérogatives aux autorités intermédiaires, un regroupement, très partiel, des hameaux et villages en communes plus grandes. La suppression de l'échelon administratif seigneurial conduisit les élites traditionnelles à investir massivement la nouvelle administration. Les activités de ces maires montrent bien qu'il s'agissait pour eux de tenter de préserver autant que possible les anciens privilèges. Mais une administration cantonale permettait une meilleure maîtrise territoriale par les partisans de l'ordre ancien. Cependant, au fil des années, et notamment après la révision des nominations en 1810, la proportion des maires nobles est retombée. Ce recul de l'emprise personnelle des anciennes élites s'observe aussi dans d'autres départements ${ }^{55}$. À la fin du règne de Jérôme, le poids numérique des nobles dans l'administration communale est faible dans l'ensemble du royaume ${ }^{56}$. Ces vicissitudes représentaient l'essentiel de l'instabilité des fonctionnaires. Dans la plupart des communes, une administration à la française ne semble pas avoir posé plus de problèmes que les administrations précédentes. Par la centralisation, le gouvernement soustrayait les collectivités locales au contrôle des élites traditionnelles et garantissait une répartition des charges plus favorable aux couches inférieures de la population.

Après la chute de l'Empire, les anciennes élites des territoires westphaliens, ayant de nouveau de bonnes relations avec le gouvernement, œuvraient en faveur de l'abolition $\mathrm{du}$ régime municipal westphalien, auquel le gouvernement prussien semblait tenir. Leurs efforts furent couronnés de succès en 1833.

Bien sûr, les « malheurs du temps » alourdissaient la tâche des administrateurs mais les répercussions sur le fonctionnement administratif semblent bien avoir été numériquement réduites et géographiquement circonscrites. Mais les problèmes liés à la guerre aussi bien que le combat entre le gouvernement et les élites provinciales pour le contrôle des collectivités furent-ils si différents des expériences qu'avaient acquises les organisateurs du royaume de Westphalie dans leur propre patrie?

\section{ANNEXES}

Glossaire des principaux termes institutionnels et de quelques catégories socioprofessionnelles

Collèges provinciaux - Administrations provinciales organisées en collège. Les chambres administratives (Domänenkammern) administraient les domaines royaux et prélevaient la contribution foncière, les Cours de justice (Regierungen) étaient l'instance d'appel pour les roturiers et la première instance pour les privilégiés, mais avaient aussi des missions religieuses et scolaires. Le collège des accises s'occupait des impôts indirects.

Kossathe - Petit exploitant agricole, appartenant à la couche intermédiaire de la population rurale, inférieure aux laboureurs, mais supérieure aux journaliers. Leurs exploitations n'avaient souvent qu'un quart, parfois un tiers de la superficie de celles 
des paysans d'un demi-attelage. Ils ne sont pas obligés de faire des corvées avec un attelage, mais les autres corvées pèsent souvent plus lourdement sur leurs petites exploitations que sur celles des laboureurs. Ces catégories de paysans servaient en général aussi à la classification fiscale. Les Kossathen exerçaient parfois des activités artisanales complémentaires.

Laboureur (Ackermann, parfois appelé Bauer) - Exploitant agricole de parfois plus d'une trentaine d'hectares disposant d'un train de labour complet. En l'absence d'un seigneur résidant ou d'un fermier seigneurial, il forme la couche sociale supérieure de la société rurale, détenant le plus souvent les principales fonctions de l'administration villageoise.

Magistrat - Organe administratif collégial des villes en Prusse, composé d'un ou de plusieurs bourgmestres, d'un camérier et de quelques conseillers.

Provinces - Unités administratives constitutives de la monarchie prussienne, de taille variable. Il s'agissait d'unités historiques, progressivement intégrées dans la monarchie. Le duché de Magdebourg était un ancien archevêché sécularisé au temps de la Réforme et cédé par la Saxe électorale au Brandebourg par le traité de 1648. Les provinces étaient subdivisées en cercles pour l'administration de la campagne. L'administrateur d'un cercle était un propriétaire noble, l'un des seigneurs regroupés dans le cercle. Les villes étaient administrées séparément.

Richter et Schulze - Noms désignant les officiers villageois (Dorfvorsteher - littéralement préposé de village), généralement nommés par le seigneur, administrant les communes rurales. En raison de la non-séparation de la justice, de l'administration et de la police, ils présidaient aussi les cours villageoises, l'échelon le plus bas de la hiérarchie judiciaire, épaulé par plusieurs jurés. La fonction de Schulze pouvait être liée à l'exploitation paysanne, mais souvent le Schulze ne bénéficiait que de l'exemption des corvées et redevances ou jouissait d'autres privilèges. Il avait certaines obligations touchant à l'organisation des corvées seigneuriales.

Villes et villages - Les villes et villages avaient des statuts différents. Les villes « immédiates » étaient directement subordonnées à la juridiction et administration royale, leurs principaux administrateurs, un collège de bourgmestres (voir magistrat), étaient nommés par le gouvernement de Berlin, les autres agents par l'administration royale de la province. Dans ses choix, la chambre administrative devait respecter un certain nombre de principes, comme celui de nommer de préférence d'anciens militaires aux emplois publics. Les villes devaient payer l'accise, l'impôt indirect, mais non la Kontribution, une sorte de contribution foncière. Celle-ci pesait sur les campagnes. Les villages étaient soumis à la juridiction seigneuriale, mais le seigneur était souvent le roi, à savoir dans tous les domaines royaux. Le gouvernement nommait les justiciers (Justizamtmänner - terme souvent traduit par baillis par les administrateurs westphaliens). Les justiciers et administrateurs des seigneuries nobiliaires et ecclésiastiques étaient nommés par les seigneurs, mais soumis, pour ce qui est de la justice, au contrôle de la cour de justice provinciale. 


\section{NOTES}

1.Le concept de la bureaucratie réformatrice a été introduit au début des années 1980 , notamment par Barbara VOGEL : Allgemeine Gewerbefreiheit. Die Reformpolitik des preussischen Staatskanzlers Hardenberg, Göttingen, Vandenhoek und Ruprecht,1983, 340 p. ; l'idée a aussi été développée par Christine VAN DEN HEUVEL, Beamtenschaft und Territorialstaat : Behördenentwicklung und Sozialstruktur der Beamtenschaft im Hochstift Osnabrück, 1500-1800, Osnabrück, H. Th. Wenner, 1984, 304 p.

2.Wolfgang Neugebauer a dû constater en 1992 que l'idée de l'État modernisateur dominait largement l'historiographie allemande : Reformen im Osten. Politischer Wandel im Osten : Ost- und Westpreußen von den alten Ständen zum Konstitutionalismus, Stuttgart, F. Steiner, 1992, 649 p., p. 12.

3.Le concept de la bureaucratie réformatrice a inspiré plusieurs thèses sur les administrateurs supérieurs de certaines principautés allemandes, comme celle de Eckhardt TREICHEL : Der Primat der Bürokratie. Bürokratischer Staat und bürokratische Elite im Herzogtum Nassau, 1806-1866, Stuttgart, F. Steiner, 1991, 649 p.

4.Ibid. p. 354.

5.Stefan BRAKENSIEK, Fürstendiener,Staatsbeamte, Bürger. Amtsführung und Lebenswelt der hessischen Ortsbeamten 1750-1830, Göttingen, Vandenhoek und Ruprecht, 1997, p. 148-150 ; l'auteur détaille les querelles entre un maire de canton westphalien plein de zèle et un maire de commune, réticent à la rigueur bureaucratique.

6.Stuart J. Woolf, Napoléon et la conquête de l'Europe, Paris, Flammarion, 1990, p. 163 : «Pour les gouvernants, les problèmes sont liés avant tout aux postes locaux, qui forment la base de la pyramide administrative. »; p. 164 : « Partout les préfets et souspréfets se plaignent de ce qu'il est impossible de trouver des candidats pour les communes rurales et les petites villes. » Selon Bettina SEVERIN-BARBoutIE, la difficulté de trouver des personnes volontaires et capables de remplir la fonction de maire aurait aussi obligé le gouvernement du grand-duché de Berg à regrouper les communes, « Neugestaltung der Verwaltung vor Ort : Die Rekrutierung der lokalen Amtsträger im Großherzogtum Berg », communication au colloque de Münster (Westphalie), Das Königreich Westfalen und das Großherzogtum Berg. Quellen, Forschungen und Deutungen, 15-16 avril 2004 (à paraître). Cependant, ce phénomène de la répugnance pour les fonctions locales dans le grand-duché de Berg n'a été nullement quantifié par l'auteur de cette communication.

7.Le point de vue de Horst DIPPEL, qui voit dans la fonction de maire un moyen d'ascension sociale attractif est plutôt exceptionnel : « Möglichkeiten und Grenzen des sozialen Aufstiegs im napoleonischen Rheinland. Die Maires des Arrondissement Köln 1801-1813 », Zeitschrift für historische Forschung 18/2 (1991), p. 215-217 ; d'ailleurs, Hartmut HARNISCH, historien de la RDA, estimait que, dans les territoires allemands, des communautés rurales autonomes en état de fonctionner avaient existé jusqu'à l'époque industrielle. Selon lui, la restriction progressive, mais incomplète, de leurs libertés avait commencé depuis le $\mathrm{XVI}^{\mathrm{e}}$ siècle et était l'œuvre de la justice seigneuriale : HARNISCH, « Landgemeinde, feudalherrlich-bäuerliche Klassenkämpfe und Agrarverfassung im Spätfeudalismus », Zeitschrift für Geschichte, 26, 1978, p. 887-897. 8.Philippe CONZE, « Die Neuordnung Deutschlands im napoleonischen System und die inneren Reformen in den deutschen Staaten », dans : Kurt G. A. JESERICH, Hans POHL, Georg Christoph UnRUH, Deutsche Verwaltungsgeschichte, Stuttgart, Deutsche 
Verlagsanstalt, vol. II, 811 p., p. 42 sq. ; Horst MATZERATH, Urbanisierung in Preußen, Stuttgart, W. Kohlhammer, 1985, 453 p., p. 29

9.Andreas SCHULZE, Herrschaft durch Verwaltung. Die Rheinbundreformen in Hessen-

Darmstadt unter Napoleon (1803-1815), Stuttgart, F. Steiner, 1991, p. 122 sq.

10.Les exemples pour ce type de proposition sont nombreux pour les départements de l'ancienne France comme pour les territoires réorganisés à la française, que ce soit sous forme de département réuni ou d'États satellite. Pour un département hanséatique, celui de l'Ems-Supérieur, dont certaines parties avaient appartenu au royaume de Westphalie : Antoinette IOULIA, « Le personnel administratif du département de l'Ems Supérieur, (1810-1813) » Bulletin d'Histoire économique et sociale, 1973, p. 140.

11.Jean-Pierre JESSENNE attire par ailleurs l'attention sur des études récentes qui nuancent le bilan négatif des municipalités cantonales : «Communautés, communes rurales et pouvoirs dans l'État napoléonien », dans Voies nouvelles pour l'histoire du Premier Empire. Territoires, Pouvoirs, Identités, textes réunis et présentés par Natalie PetiteAu, Paris, La boutique de l'histoire, 2003, p. 163.

12.André Tinon, « La fusion des communes dans le département de la Dyle sous le régime napoléonien », Revue Belge de Philologie et d'Histoire, 1965, p.534 sq.

13.NAPOLÉON Ier, Correspondance de Napoléon, $n^{\circ}$ 13361, lettre du 15 novembre 1807.

14.Landeshauptarchiv von Sachsen-Anhalt - Magdeburg (Archives du Land de SaxeAnhalt - dépôt de Magdebourg, par la suite : LHSAM), Rep. A9, a, VI, Magdeburg, n 15, vol. VI, pétition du 7 avril 1801 et la réponse de la chambre de Magdebourg du 17 avril 1801.

15.Ibid. Rep. A 9, a, VI, Schoenebeck, $\mathrm{n}^{\circ} 15$, Instruction pour le conseil municipal de la ville de Schönebeck, 1er septembre 1783.

16.Landeshauptarchiv von Sachsen-Anhalt Außenstelle Wernigerode (Archives du Land de Saxe-Anhalt, dépôt de Wernigerode, par la suite LHSAW), Rep.E von der Schulenbourg, $n^{\circ}$ 59/4, Règlement administratif du royaume de Westphalie, 1808.

17.Ibid., article 43.

18.Ibid., articles 37-42.

19.Ibid., Rep. B 19 d, nº 93, 13 : circulaire ministérielle du 21 mars 1809.

20.Ibid., Rep.B18, II, n² 273, I.

21.Archives du ministère des Affaires étrangères (par la suite AAE), Correspondance politique, Westphalie, vol. 14, 158.

22.LHSAW, Rep. B 19, d, n 93, circulaire préfectorale adressée aux maires.

23.Ibid. Rep. B 18 II, $n^{\circ} 1$, a-d, 135 des 531 communes ; il est probable que la proportion fut supérieure, la documentation étant peut-être lacunaire.

24.LHSAM, Rep. A 7, $\mathrm{n}^{\circ} 81$.

25.LHSAW, Rep. H Erxleben, $n^{\circ} 3624$

26.LHSAM, Rep. A 8, $\mathrm{n}^{\circ}$ 92, « Liste tarifaire pour les sportules des conseillers provinciaux et des villes du duché de Magdebourg ", 1786, la révision pouvait donc être assurée aussi par les conseillers.

27. Bibliothèque Nationale de Saint-Pétersbourg, Fonds 993, Archives Westphaliennes, carton $n^{\circ} 3$, pièce 350 .

28.LHSAW, Rep./B18/II/, no 19/Id, 23, lettre du sous-préfet de Salzwedel au préfet, en date du 24 octobre 1812.

29.AAE, Correspondance politique, Westphalie, vol. 14, 162, tableau IX. 30.LHSAW, Rep./B18, I, n 267a, listes des candidats pour le poste de conseiller municipal. 
31.Gustav ANTON VON WOLFRADT, « Denkwürdigkeiten eines ehemaligen Braunschweigischen Ministers », dans Deutsche Rundschau, XLVI (1886), p. 66. 32.LHSAW, Rep. B 18, I, n² 285, 19, lettre du 25 septembre 1809 au préfet de l'Elbe. 33.LHSAM, Rep. A8 $n^{\circ} 77$ i et j ; Geheimes Staatsarchiv Preussischer Kulturbesitz Berlin (Archives de la Prusse par la suite GSTPK) Rep. B 4, n 63-66, dossiers personnels, 1807.

34.LHSAW, Rep. B35, II, e, no. 7: dans le département du Harz, le nombre de postes vacants après la première vague de nominations est de 7 pour 236 communes, soit moins de $3 \%$.

35.Ibid., Rep. H Erxleben II, no.3624, 69.

36.LHSAM, Rep. A 8, $n^{\circ}$ 57, vol. II, 134 : plainte des laboureurs du village de Kamern, à l'est de l'Elbe contre la nomination d'un petit paysan par les autorités de la seigneurie royale, novembre 1766 ; Rep. A 9, c, III, R, n 41, 8, plainte des laboureurs du village de Nordgermersleben contre la nomination du petit paysan Schröder par les autorités de la seigneurie royale d'Alvensleben, 18 juillet 1795.

37.Archives municipales de Schönebeck, Chronique (non publiée) Walter Schulze, « Aus der Geschichte der Stadt Schönebeck », p. 531 sq.

38.LHSAW, Rep. B18, I, no.283, I, 10.

39.Ibid., Rep. B 18, I, n 273, I, 94.

40.Ibid., Rep. B18, I, n 383, I, 93, lettre d'Angern au préfet en date du 13 août 1808 ; les historiens de la seigneurie domaniale ont montré que les laboureurs entretenaient effectivement plus de chevaux que ne l'aurait exigé le fonctionnement de leurs exploitations et ceci en raison des corvées dues au seigneur.

41.Ibid., Rep. B18, I, n 383, I, 97, réponse du préfet le 31 août 1808.

42.Ibid., Rep. B 18, I, n² 279, vol. II, 306.

43.Ibid., Rep. B18, II, $\mathrm{n}^{\circ} 1$.

44.Ibid. Rep. C 4, A, 1, n 64, liste des agents publics, de leurs traitements et de leurs indemnités pour les frais de bureaux, mars 1814 .

45.Ibid., Rep. B18, I, n² 283, vol. I, 81 ; c'était l'une des rares communes où les différentes catégories n'étaient pas représentées au conseil municipal, qui était dominé par les laboureurs.

46.Ainsi, les autorités supérieures rejettent le plan de répartition qui prévoit de taxer également les villageois dépourvus de propriétés foncières : Ibid., Rep. B18, II, n²1, vol. I, b, budget pour 1813 .

47.GSTAPK, königreich Wertfalen, Rep. 3, le préfet au ministre, le 8 août 1813.

48.LHSAW, Rep.B18, II, $\mathrm{n}^{\circ}$ 19, III, f, 1, lettre du maire de canton du 18 décembre 1813, de Hyronimusville.

49.Déjà Franz Mehring souligne que le chef de la garnison était le maître absolu dans la ville. Personne n'était à l'abri de ses exactions : « Die preußische Städteordnung, 1808 », dans Zur Geschichte Preußens, Berlin, Dietz, 1984, p. 145, réédition d'un article de 1912. 50.Archives municipales de Schönebeck, série Frohse, II, 11, tableau de la population. 51.LHSAM, Rep. A, 9, VI, $n^{\circ} 15$, correspondance très volumineuse entre les autorités militaires, la chambre provinciale et l'administration municipale de Schönebeck, 1772. 52.Ibid., Rep. A 7, $\mathrm{n}^{\circ} 81$.

53.Ibid., A 9 c, VII, lettre $\mathrm{R}, \mathrm{n}^{\circ}$ 19, 26 janvier 1776, pétition signée par tous les membres de la commune.

54.Ibid., Rep. C Wolmirstedt, $\mathrm{n}^{\circ}$ 67, Inventaire des écrits, livres et autres objets appartenant au Schulzenamt, de 1874 ; parmi ces écrits, on trouve le bulletin des lois à 
partir de 1817, les circulaires à partir de 1816, les annonces publiques depuis 1817 , les collections de textes de lois à destination des fonctionnaires de 1806 à 1836.

55.LHSAW, Rep. B 26, 2, n 30 (1808) et AN 400AP/94 (1812). Dans les deux districts de Halberstadt et de Halle appartenant au département voisin de la Saale, le nombre de maires nobles passe de 24 à 12 pour au total 210 communes.

56.AN 400AP/94, Nous avons pu dénombrer le nombre de maires nobles dans sept des huit départements en 1812. À ce moment il y a 62 maires nobles sur 2919 communes mais 41 nobles sont maires de cantons pour 270 maires de canton au total.

\section{RÉSUMÉS}

Après la victoire sur la quatrième coalition, Napoléon forma le royaume de Westphalie à partir de certaines des provinces conquises. Le modèle administratif français fut introduit dans cet Étatsatellite. Parmi les changements institutionnels imposés aux territoires conquis, les réformes de l'administration communale furent souvent l'objet de critiques. Nous nous attachons à examiner de près les modifications des institutions, des enjeux sociaux et du fonctionnement de l'administration communale imposées aux royaume de Westphalie à l'exemple d'un de ses départements, celui de l'Elbe. Nous cherchons à montrer que les critiques sur le fonctionnement administratif cachaient souvent des enjeux sociaux; la réalisation des réformes administratives était donc intrinsèquement liée à l'héritage révolutionnaire.

Local administration in the Kingdom of Westphalia. After the victory of the Fourth Coalition, Napoleon formed the Kingdom of Westphalia from some of his conquered provinces. The French administrative model was introduced in this Satellite State. Among the institutional changes imposed on the conquered territories, the reforms in local administration were often the subject of criticism. The author examines closely the modifications in institutions, the social issues, and the functioning of local administration of the Kingdom of Westphalia in one of its departments: the Elbe. He shows that criticisms of administrative operations often concealed social issues; hence, the accomplishment of administrative reforms was intrinsically tied to the Revolutionary heritage.

\section{INDEX}

Mots-clés : royaume de Westphalie, Prusse, administration communale, municipalités cantonales, réformes napoléoniennes, réaction seigneuriale

\section{AUTEUR}

\section{NICOLA PETER TODOROV}

37, boulevard de Verdun, 76000 Rouen, nicola.todorov@wanadoo.fr 